\title{
Stability of sucrose drinking curves in genetically heterogeneous mice
}

RALPH L. LEVINE, DEPARTMENT OF PSYCHOLOGY, MICHIGAN STATE UNIVERSITY, East Lansing, Mich. 48823

Fifteen genetically heterogeneous mice were given a simultaneous choice of five sugar concentrations: $0 \%$ (tap water), $15 \%$, $30 \%, 45 \%$, and $60 \%$ sucrose. Individual differences in sucrose drinking accounted for $60 \%$ of the total variability in the experiment, and sucrose patterns for individual Ss neither corresponded to each other nor to the mean curve. These massive differences in drinking patterns proved to be stable and reliable over time so that an "error" model, which depends solely upon random fluctuations around the mean curve, seems untenable.

A continuing controversy in the field of experimental psychology concerns the adequacy of the mean curve to represent the behavior of organisms (Sidman, 1952; Estes, 1956; Tucker, 1966). Although most of the arguments have centered around problems of learning, the controversy might apply to other areas of psychology such as sucrose preference behavior. In this field most of the data are reported in the form of mean intake curves occasionally including the standard deviations around each mean point.

If the extent and magnitude of individual curves are of some importance in understanding sucrose preference behavior, then they must be studied in addition to the mean curve. On the other hand, some investigators (e.g., Jacobs \& Scott, 1957; Carpenter, 1958) have even placed a number of $S$ s together in the same testing apparatus to measure the total group preference function. When testing with this procedure, however, one must make the a priori assumption that the total intake obtained in this manner is equivalent to the operation of summing individual preference curves measured under separate conditions. Even if such an assumption of linearity were true empirically, the role of individual differences in sucrose preference would be obscured by such group procedures.

In mice the magnitude of variation in sucrose preference may depend upon genetic parameters as well as environmental factors. Levine (1965) found minimal variability within five inbred strains of mice compared to the behavioral variability of genetically heterogeneous animals formed from an appropriate crossing of four of these strains. For the inbreds the mean curve adequately summarized the performance of individuals in the experiment. This was not the case for the heterogeneous population.

The use of the mean curves to represent the pattern of sucrose drinking seems more appropriate when differences in the curves are mainly determined by random fluctuations in many small variables during the experiment. If the differences in patterns were highly stable over time, i.e., each S's curve was reliably different from other curves, an assumption of random fluctuations in the experiment would not be enough to account for such reliability.

The problem of the present experiment was to test the adequacy of using the mean curve as an index of sucrose preference behavior. This was done by examining the characteristics and reliability of individual profiles sampled from a population of genetically heterogeneous mice.

Method. Sixteen naive mice, half of which were males were taken from a four-way cross population formed from mating four inbred strains of mice: A/Crgl, Rlll/Crgl, C57B 1/Crgl, and DBA/Crgl. During the experiment, one of the females died. The analysis will therefore be based upon 15 Ss whose ages ranged from 94 to 160 days at the start of the experiment.

Five different sucrose concentrations were simultaneously presented to the Ss: $0 \%, 15 \%, 30 \%, 45 \%$, and $60 \%$ (weight/ volume). To counteract the effect of position preference, a total of 45 different orders of concentrations were assigned randomly to protocol sheets, so that each animal received three different orders of solutions.
On the first day of the experiment, after being weighed, the Ss were placed upon the testing rack, the five graduated cylinders fitted with metal tips and rubber stoppers were presented to the $S$, and the level of each solution was measured to the nearest $.1 \mathrm{ml}$. In addition, laboratory chow was always available throughout the experiment.

Each order of concentrations assigned to the $S$ remained in the cage for three days before changing positions of the bottles to a new order. Daily readings of total intake per solution were taken, and when necessary the cylinders were refilled with fresh solutions. Upon the ninth day, the animals were again weighed and removed from the testing situation.

Results. The group "incentive" curve, in terms of the total liquid intake measured over the nine day period, was 9.9, 21.6, $15.3,9.9$, and $9.9 \mathrm{ml}$ for the $0 \%, 15 \%, 30 \%, 45 \%$, and $60 \%$ sucrose solutions respectively. An analysis of variance revealed that both main effects, Subjects $(F=3.1 ; \mathrm{df}=14 / 150 ; \mathrm{p}<.01)$ and Concentrations $(F=2.96 ; \mathrm{df}=4 / 56 ; \mathrm{p}<.05)$, as well as the interaction between Subjects and Concentrations $(F=8.4$; $\mathrm{df}=56 / 150 ; p<.01$ ) were significant. The proportion of sum of squares (SS interaction/SS total), an index of relative variability, for this interaction was .62 , which was by far the most important source of variation.

Figure 1 shows 12 of the 15 individual curves. The others not shown varied in the general level of intake and in shape so that they could not be placed conveniently in the figure. It can be seen that there is a great deal of difference among these curves. An analysis of rank ordering of concentrations for each $S$ revealed that none of the 15 curves corresponded to each other and in fact no curve corresponded to the overall mean function.

With such idiosyncratic data it is imperative to investigate the reliability of these findings. The approach used was as follows. Every S's curve was analyzed separately to see whether the rank order of his preferences changed significantly with time. Since a large number of bottles were presented to the animals, the information obtained for three consecutive days under a single order of presentation was pooled. This was done because the animals spread their drinking among all five bottles so that one day's observation could not provide an adequate measure of preference. Measures of intra-S reliability were obtained for each individual by means of Kendall's coefficient of concordance, $W$, which measures the agreement among the three sets of rankings for each S (Siegel, 1956). Eleven animals were significantly reliable at least at the $5 \%$ level. Thus for this situation, the Ss in general have relatively stable preferences.

Discussion. The results of sucrose preference experiments have been commonly reported in the form of mean curves which, in theory, represent the "typical pattern" found in the group.

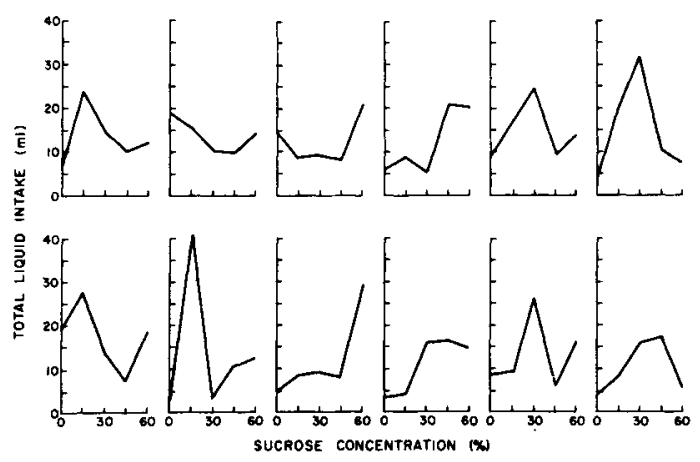

Fig. 1. Twelve individual intake curves for concentrations of sucrose presented simultaneously over a nine day period. 
Unfortunately, for the genetically heterogeneous mice used in this study, individual differences in the shape of the intake curve were far too great to represent preference by the mean function. One curve simply does not represent what happened in the experiment.

Average intake curves, without reference to individual variability in drinking patterns, may lead to an over-simplified and inaccurate description of sucrose drinking behavior. In the present experiment, for example, the mean intake curve happens to be shaped in the form of an inverted $U$. It seems natural to utilize this familiar shape in describing sucrose drinking behavior, but a model based upon the assumption that individual curves were of this form might in all probability be doomed to failure.

The results showed that the individual differences in sucrose drinking patterns were for the most part stable differences. This reliability in the preference curves casts doubt upon the view that individual differences in patterns are caused merely by random fluctuations of a large number of extraneous variables. Such a theory of random variability around the mean pattern would have difficulty explaining how a particular $S$ managed to show the same basic curve three times in a row. This massive, stable interaction between Ss and concentrations bears further study in future experiments of sucrose preference.

\section{REFERENCES}

CARPENTER, J. A. A comparison of stimulus-presentation procedures in taste-preference experiments. J. comp. physiol. Psychol., 1958, 51, 561-564.

ESTES, W. K. The problem of inference from curves based upon group data. Psychol Bull., 1956, 53, 134-140.

JACOBS, H. L., \& SCOTT, M. L. Factors mediating food and liquid intake in chickens: 1. Studies on preference for sucrose or saccharine solutions. Poultry Sci, 1957, 36, 8-15.

LEVINE, R. L. Genotypic and environmental components of sucrose drinking behavior: the relationship between measurement procedures and individual differences in preference. Unpublished doctoral dissertation, University of California, Berkeley, 1965.

SIDMAN, M. A note on functional relations obtained from group data. Psychol Bull., 1952, 49, 263-273.

SIEGEL, S. Nonparametric methods for the behavioral sciences. New York: McGraw-Hill, 1956.

TUCKER, L. R. Learning theory and multivariate experiment: lllustration by determination of generalized learning curves. In R. B. Cattell (Ed.), Handbook of multivariate experimental psychology. Chicago: Rand McNally Company, 1966. Pp. 476-501. 